\title{
Prevalence of Scoliosis in Hypermobile Ehlers-Danlos Syndrome
}

\author{
Authors: \\ Fabrice Gillas, ${ }^{1}$ Ahmed Mekki, ${ }^{2}$ Malika Foy, ${ }^{1}$ Robert Carlier, ${ }^{2,3}$ * Karelle \\ Benistan ${ }^{1,3}$ \\ 1. Department for Non-vascular Ehlers-Danlos Syndromes, Raymond Poincaré \\ Hospital, Garches, France \\ 2. DMU Smart Imaging, Department of Radiology, Raymond Poincaré Hospital, \\ Garches, France \\ 3. INSERM U1179, Versailles Saint-Quentin-en-Yvelines University, Montigny-le- \\ Bretonneux, France \\ *Correspondence to karelle.benistan@aphp.fr
}

Disclosure:

The authors have declared no conflicts of interest.

Acknowledgements: The authors would like to thank the Ehlers-Danlos Society for allowing use of the checklist of 2017 diagnostic criteria for hypermobile Ehlers-Danlos syndrome; and to thank Frédéric Barbot for his advice.

Received:

17.12 .20

Accepted:

22.01.21

Keywords:

Connective tissue disorders, hypermobile Ehlers-Danlos syndrome (hEDS), joint hypermobility, scoliosis.

Citation:

EMJ. 2021;6[2]:54-62.

Abstract

Objective: The main purpose of this study was to evaluate the prevalence, form, and severity of scoliosis in a population of adults meeting the 2017 criteria for hypermobile Ehlers-Danlos syndrome (hEDS). The second objective was to compare the prevalence of scoliosis versus other criteria at initial hEDS diagnosis.

Methods: A retrospective study looking at the frequency and severity of scoliosis in adults ( $N=28)$ meeting the 2017 diagnostic criteria for hEDS through analysis of a full spine EOS $^{\circledR}$ X-ray (EOS imaging, Paris, France) performed at the initial diagnosis. Severity was defined by the Cobb angle.

Results: At the initial diagnosis, the mean age was 30.1 years (standard deviation [SD]: \pm 10.18 years). Twenty-nine percent $(n=8 / 28)$ of patients fulfilling hEDS criteria presented with scoliosis. Thirty-two percent $(n=9 / 28)$ of patients had scoliotic inflection and $39 \%(n=11 / 28)$ had no scoliosis. Scoliosis was mild-to-moderate with a mean Cobb angle of $13.6^{\circ}\left(\mathrm{SD}: \pm 3.5^{\circ}\right.$ ). None of the patients had severe scoliosis requiring surgery. Compared to the 2017 diagnostic criteria, it is noteworthy that scoliosis prevalence in this present study population ranks at the level of the most frequent ones.

Conclusion: This study provides interesting information regarding frequency of scoliosis and scoliotic inflection in a group of patients with hEDS. Although the patients did not present with severe forms of scoliosis at initial diagnosis, the results highlight the importance of systematically looking for scoliosis in adult and young patients, in order to follow progression and ensure appropriate management. 


\section{INTRODUCTION}

Joint hypermobility is often asymptomatic. It can sometimes progress to joint instability, causing sprains, dislocations, or pain suggestive of EhlersDanlos syndrome (EDS). Joint hypermobility can also be associated with other pathologies, such as neuromuscular diseases, Marfanoid syndromes, or skeletal dysplasia. ${ }^{1}$ The diagnostic process for symptomatic joint hypermobility must be rigorous and structured to guide the diagnosis.

EDS are a heterogeneous group of inherited connective tissue disorders caused by different mutations in genes involved in the structure or biosynthesis of collagens and extracellular matrix proteins. ${ }^{2}$ The collagens involved are Types I, III, V, and XII. ${ }^{2}$ Some EDS are the result of abnormalities in the synthesis of glycosaminoglycans. ${ }^{3}$ There are currently 14 types of EDS. ${ }^{2,4}$ The phenotypic hallmarks include joint hypermobility, skin hyperextensibility, and tissue fragility. The prognosis and management of each type of EDS are different, requiring precise clinical diagnosis and genetic confirmation. EDS are rare diseases, with an estimated global frequency of one in $5,000 .{ }^{5}$ Inheritance is most often autosomal dominant but with variable expression in the same family. ${ }^{3}$ Hypermobile EDS (hEDS) is the most common type. ${ }^{6}$ It mainly affects women. ${ }^{7}$ Its diagnosis remains clinical, in the absence of clearly identified molecular bases. An interview on personal and family clinical history, followed by a rigorous clinical examination using a checklist of diagnosis criteria, confirms the diagnosis. ${ }^{2}$ It is cautious and useful, especially with young patients and adolescents, to reassess the patient several times before confirming the diagnosis of hEDS.

Scoliosis is a structural deformity of the spine in the three dimensions of space. The diagnosis is confirmed when the Cobb angle is $10^{\circ}$ or higher and axial rotation is recognised on the X-ray. It should not be confused with a scoliosis attitude, which originates outside the spine (such as in length inequality of the lower limbs or pelvic rotation) and is totally reducible when patients lie down. It normally does not progress to scoliosis. ${ }^{8}$

Twenty percent of scoliosis are not idiopathic and may be linked to an underlying disease with hypermobility; as a consequence, it was one of the clinical criteria discussed but not included during the establishment of the new 2017 criteria for hEDS. ${ }^{9}$

Limited data exist in the literature regarding the prevalence and severity of scoliosis in EDS and, in particular, in hEDS. ${ }^{10-12}$ The main objective of this study was to assess the prevalence, form, and severity of scoliosis in hEDS through a retrospective study in a homogeneous population of adults fulfilling the 2017 criteria for hEDS. The second objective was to compare the prevalence of scoliosis relative to the prevalence of other clinical features at diagnosis.

\section{METHODS}

\section{Patient Selection}

This was a retrospective study based on the analysis of medical records of patients who visited the reference centre of rare diseases between 2017 and 2020. Twenty-eight patients were selected. Eligibility criteria included patients aged $\geq 18$ years, fulfilling the 2017 criteria for hEDS, and who had a full spine X-ray at the time of diagnosis. 2,13 Pregnant patients and those without full spine X-ray available were not included in the study.

\section{Diagnostic Procedures for hEDS and Evaluation of Generalised Joint Hypermobility}

Each patient had been clinically examined by an expert physician from the reference centre of rare diseases to confirm hEDS diagnosis. The 2017 diagnostic criteria were evaluated to confirm the diagnosis, following the checklist provided by the international EDS consortium. ${ }^{13}$

During the examination, patients underwent a generalised joint hypermobility measure using the Beighton score.1,14,15 Each hypermobile joint scores one point, making it possible to define a total score out of 9 . The positivity threshold must be adapted according to age.16 Authors consider generalised joint hypermobility with a score of 5 out of 9 for men and women $<50$ years old, and 4 out of 9 in adults over $>50$ years old; in prepubertal children and adolescents, the threshold is 6 out of 9 (Box 1A). The interobserver reproducibility is rather good (kappa: 0.80). ${ }^{17}$ Because joint laxity decreases with age in the 
general population and in hypermobile patients, the Hakim and Grahame simplified questionnaire (five-part questionnaire) was also used to evaluate history of hypermobility. ${ }^{1}$ Positive response to two or more questions between five items brings an additional point to the measured Beighton score (Box 1B). The Beighton score and the Hakim and Grahame simplified questionnaire are two criteria of the 2017 criteria for hEDS.

An objective of clinical examination was also to eliminate other connective tissue disorders (e.g., other types of EDS, Marfan syndrome, and LoeysDietz syndrome). Moreover, according to 2017 recommendations, all the patients underwent a cardiac ultrasound to evaluate both the mitral valve and aortic root. ${ }^{2}$

\section{Detection of Scoliosis Using Full Spine X-ray}

During the measure of the Beighton score, the authors evaluated the ability for patients to put their hands flat on the floor with knees extended; this evaluation would unmask the presence of thoracic or lumbar scoliosis. After checking for pregnancy, patients with hEDS benefited from a complete spine radiographic examination using the EOS $^{\circledR}$ method (EOS imaging, Paris, France). The EOS methodology uses a radiation dose 10-times lower than conventional radiology techniques, 1,000-times lower than that of a scanner, and optimises the follow-up and management of patients with osteoarticular pathologies of the spine and lower limbs (e.g., scoliosis and static disorders). The reconstructions in two- or three-dimensions, with lateral, frontal, and horizontal views, allow a very precise analysis of the deformities of the spine, from the head to the pelvis. This system is also of interest in terms of therapeutic monitoring.

\section{Box 1: Beighton score and five-part questionnaire for generalised joint hypermobility.}

A

\begin{tabular}{|l|l|}
\hline Dorsiflexion of the $5^{\text {th }}$ metacarpophalangeal joint to $>90^{\circ}$ & 1 point for right, 1 point for left \\
\hline Opposition of the thumb to the volar aspect of the forearm & 1 point for right, 1 point for left \\
\hline Hyperextension of the elbows to $>10^{\circ}$ & 1 point for right, 1 point for left \\
\hline Hyperextension of the knees to $>10^{\circ}$ & 1 point for right, 1 point for left \\
\hline Ability to place hands flat on the floor with knees fully extended & 1 point \\
\hline Total score & $/ 9$ \\
\hline
\end{tabular}

B

\begin{tabular}{|l|l|l|}
\hline $\begin{array}{l}\text { Can you now or could you ever touch your hands flat on } \\
\text { the floor without bending your knees? }\end{array}$ & Yes & No \\
\hline $\begin{array}{l}\text { Can you now or could you ever bend your thumb to touch } \\
\text { your forearm? }\end{array}$ & Yes & No \\
\hline $\begin{array}{l}\text { As a child, did you amuse your friends by contort-ing your } \\
\text { body into strange shapes or could you do the splits? }\end{array}$ & Yes & No \\
\hline $\begin{array}{l}\text { As a child or teenager, did your shoulder or knee-cap } \\
\text { dislocate on more than one occasion? }\end{array}$ & Yes & No \\
\hline Do you consider yourself 'double jointed'? & Yes & No \\
\hline
\end{tabular}

A) Beighton score for generalised joint hypermobility measure. A positive Beighton score is $\geq 5 / 9$ for adults; $\geq 4 / 9$ for adults over the age of 50 years; and $\geq 6 / 9$ points for children and adolescents. B) Five-part questionnaire for generalised joint hypermobility. A positive response to two or more questions brings one additional point to the Beighton score. 
The EOS report included the measurement of sub-pelvic parameters (pelvic incidence, sacral slope, and pelvic version), the existence of disorders of the frontal or sagittal balance of the spine, the existence of a scoliotic inflection, scoliosis, its type (thoracic, thoracolumbar, or lumbar), and the Cobb angle measured for each curvature. Other spinal abnormalities such as spondylolisthesis, history of Scheuermann's disease, and vertebral abnormalities could also be detected. A classification of morphotypes defined by Roussouly in 2005 allows different types of sagittal profiles to be identified. ${ }^{18}$ Of interest in this classification (which has only been validated in adults) is its ability to understand degenerative disorders of the spine. Morphotypes with low pelvic incidence (Type 1 and 2) will be more prone to degenerative pathologies of the disc and pain, while morphotypes with high pelvic incidence (Type 3 and 4 ) will be more at-risk of degenerative slips such as spondylolisthesis.

\section{Statistical Analysis}

The characteristics of the population (e.g., sociodemographic) were described using means \pm standard deviation (SD) for continuous variables, and frequency for qualitative variables.

\section{Ethical Considerations}

Retrospective registry-based studies do not require ethics committee approval under French law. Patient data were extracted from the patient electronic record from the reference centre of rare diseases. Patient data were then collected in a database approved by the National Commission for Data Protection and Liberties (CNIL).

\section{RESULTS}

\section{Patient Characteristics}

Twenty-eight patients (27 female and one male) with a diagnosis of hEDS were selected and underwent a complete spine X-ray using EOS method and analysis (Table 1). The mean age at diagnosis was 30.10 (SD: \pm 10.18 years; range 19.00-50.00 years). The mean BMI was 25.19 (SD: $\pm 4.88)$. Seventy-one percent $(n=20 / 28)$ were employed at the time of diagnosis. Twenty-one percent $(n=6 / 28)$ had at least one orthopedic surgery at the time of diagnosis and $7 \%(n=2 / 28)$ had three or more surgeries.

\section{Prevalence of the 2017 Criteria for hEDS in the Patient Population}

This study evaluated retrospectively the prevalence of the 2017 diagnostic criteria, which made it possible to confirm the diagnosis of hEDS. Prevalence of these 2017 criteria in the patient population are detailed in Table 2 . Generalised joint hypermobility was found in $100.0 \%$ of patients. The mean Beighton score was 6/9 (SD: \pm 2 ). Sixty-four percent of patients were able to place their hands completely flat on the floor with their knees extended; $7.1 \%$ had done so in the past. Other clinical signs were found: bilateral piezogenic papules of the heels $(53.6 \%$ of patients); moderate skin hyperextensibility (46.4\%); unusually soft and velvety skin (42.9\%); large unexplained striae (39.3\%); arachnodactyly (25.0\%); and a bilateral thumb sign (Steinberg's sign) and bilateral wrist sign (Walker's sign) in $17.9 \%$ and $7.1 \%$ of patients, respectively. Furthermore, $39.3 \%$ of patients presented with swan-neck deformities of the fingers, $17.9 \%$ presented with dental crowding and/or a high or narrow palate, and $14.3 \%$ presented with atrophic scars in at least two places. One patient (3.5\%) presented with aortic root dilation and two patients (7.1\%) presented with mitral valve prolapse on their ultrasound. One patient (3.5\%) had an arm-span-to-height ratio $\geq 1.05$ and one patient $(3.5 \%)$ presented with recurrent and multiple abdominal hernias. No patients had pelvic, rectal, or uterine prolapse in their history (Table 2). Family history was found in 50.0\% of patients.

\section{Prevalence and Types of Scoliosis in the Patient Population}

In this patient population, scoliosis was found in almost $29 \%(n=8 / 28)$. None of these patients had undergone spine surgery (Table 1). Among the $29 \%$ of patients with scoliosis, $12.5 \%(n=1 / 8)$ had a right thoracic form, $12.5 \%(n=1 / 8)$ had a left thoracic form, 50.0\% $(n=4 / 8)$ had a thoracolumbar form, and $25.0 \%(n=2 / 8)$ had a unique lumbar form. A mild form of scoliosis with Cobb angle $\leq 19^{\circ}$ was found in $87.5 \%$ of patients $(n=7 / 8), 12.5 \% \quad(n=1 / 8)$ had a moderate form (Cobb angle $<29^{\circ}$ ), and none had a severe form (Cobb angle $>30^{\circ}$ ). The mean Cobb angle was $13.6^{\circ}\left(\mathrm{SD}: \pm 3.5^{\circ}\right.$ ). 
Table 1: Patient characteristics, demographic and spinal features, and EOS parameters.

\begin{tabular}{|c|c|c|c|c|c|c|c|c|c|}
\hline Age, sex & BMI & BS & $\begin{array}{l}\text { Ability to put } \\
\text { hands flat on } \\
\text { the floor }\end{array}$ & $\mathrm{PI}$ & ss & PT & $\begin{array}{l}\text { Roussouly } \\
\text { type }\end{array}$ & Type of scoliosis & $\begin{array}{l}\text { Cobb } \\
\text { angle }\end{array}$ \\
\hline \multicolumn{10}{|c|}{ Patients with scoliosis } \\
\hline $28, F$ & 24.46 & 6 & No & 48 & 32 & 16 & 1 or 2 & Left lumbar L1-L4 & $12^{\circ}$ \\
\hline $25, \mathrm{~F}$ & 23.19 & 5 & Yes & 45 & 38 & 4 & 3 & $\begin{array}{l}\text { Left thoracic } \\
\text { T1-T6 }\end{array}$ & $10^{\circ}$ \\
\hline $49, \mathrm{~F}$ & 31.63 & 7 & Yes & 75 & 42 & 33 & 3 & Right lumbar & $10^{\circ}$ \\
\hline $25, \mathrm{~F}$ & 22.20 & 7 & Yes & 53 & 45 & 8 & 3 & Right thoracic & $13^{\circ}$ \\
\hline $21, \mathrm{~F}$ & 21.20 & 6 & No & NA & NA & NA & NA & $\begin{array}{l}\text { Left } \\
\text { thoracolumbar }\end{array}$ & $15^{\circ}$ \\
\hline $36, F$ & 32.47 & 6 & Yes & 89 & 37 & 52 & 3 & Thoracolumbar & $13^{\circ}$ \\
\hline $20, F$ & 19.71 & 5 & Yes & 49 & NA & NA & NA & $\begin{array}{l}\text { Thoracic and } \\
\text { lumbar }\end{array}$ & $\begin{array}{l}15-16 \text { and } \\
22^{\circ}\end{array}$ \\
\hline $26, \mathrm{~F}$ & 17.80 & 4 & Yes & 32 & 35 & 4 & 3 & Thoracolumbar & $14^{\circ}$ \\
\hline \multicolumn{10}{|c|}{ Patients with scoliotic inflection } \\
\hline $27, \mathrm{~F}$ & 22.99 & 5 & Yes & 40 & 33 & 6 & 1 or 2 & $\begin{array}{l}\text { Right thoracic, } \\
\text { left lumbar }\end{array}$ & NA \\
\hline $33, \mathrm{~F}$ & 31.25 & 6 & NA & 44 & 34 & 10 & 1 or 2 & $\begin{array}{l}\text { Left } \\
\text { thoracolumbar }\end{array}$ & NA \\
\hline $27, \mathrm{~F}$ & 22.06 & 5.5 & Yes & 62 & 55 & 6 & 4 & $\begin{array}{l}\text { Left } \\
\text { cervicothoracic }\end{array}$ & NA \\
\hline 19, F & 23.15 & 6.5 & Yes & 56 & 51 & 5 & 4 & $\begin{array}{l}\text { Left } \\
\text { thoracolumbar }\end{array}$ & NA \\
\hline $26, \mathrm{~F}$ & 25.35 & 8 & Yes & 38 & 25 & 12 & 1 or 2 & NA & NA \\
\hline $20, \mathrm{~F}$ & 35.56 & 8 & Yes & 43 & 35 & 9 & 3 & Left lumbar & NA \\
\hline $24, F$ & 25.39 & 5 & Yes & 49 & 33 & 16 & 1 or 2 & Right lumbar & NA \\
\hline $28, F$ & 19.23 & 7 & Yes & 76 & 53 & 23 & 4 & Left lumbar & NA \\
\hline 47, F & 23.80 & 5.5 & Yes & 52 & 17 & 35 & 1 or 2 & NA & NA \\
\hline \multicolumn{10}{|c|}{ Patients without spinal abnormalities } \\
\hline $21, \mathrm{~F}$ & 24.51 & 8 & No & 27 & 28 & -1 & 1 or 2 & NA & NA \\
\hline $41, F$ & 26.40 & 8 & Yes & 55 & 43 & 13 & 3 & NA & NA \\
\hline $50, \mathrm{~F}$ & 26.26 & 4 & Yes & 51 & 41 & 11 & 3 & NA & NA \\
\hline $26, M$ & 35.24 & 7 & No & 47 & 22 & 25 & 1 or 2 & NA & NA \\
\hline $22, \mathrm{~F}$ & 30.49 & 5 & Yes & 63 & 61 & 2 & 4 & NA & NA \\
\hline $48, F$ & 31.59 & 9 & Yes & 38 & 37 & 2 & 3 & NA & NA \\
\hline $47, \mathrm{~F}$ & 26.31 & 5.5 & Yes & 62 & 37 & 26 & 4 & NA & NA \\
\hline $25, \mathrm{~F}$ & 22.84 & 5 & Yes & 37 & 26 & 11 & 1 or 2 & NA & NA \\
\hline $21, F$ & 19,95 & 8 & Yes & 48 & 27 & 21 & 3 & NA & NA \\
\hline $42, \mathrm{~F}$ & 20,45 & 6 & Yes & 50 & 48 & 2 & 4 & NA & NA \\
\hline $19, \mathrm{~F}$ & 19,88 & 6 & No & 36 & 30 & 6 & 1 or 2 & NA & NA \\
\hline
\end{tabular}

BS: Beighton score; EOS: EOS ${ }^{\circledR}$ X-ray (EOS imaging, Paris, France); F: female; M: male; NA: not assessed; Pl: pelvic incidence; PT: pelvic tilt; SS: sacral slope. 
Table 2: Prevalence of the 2017 diagnostic criteria for hypermobile Ehlers-Danlos syndrome and scoliosis in the patient population.

\begin{tabular}{|c|c|}
\hline 2017 criteria for hypermobile Ehlers-Danlos syndrome & Frequency \\
\hline Generalised joint hyperlaxity & $100.0 \%$ \\
\hline Bilateral piezogenic papules of the heels & $54.0 \%$ \\
\hline Positive family history & $50.0 \%$ \\
\hline Moderate skin hyperextensibility & $46.0 \%$ \\
\hline Unusually soft and velvety skin & $43.0 \%$ \\
\hline Large unexplained striae & $39.0 \%$ \\
\hline Swan-neck deformities of the fingers & $39.0 \%$ \\
\hline Arachnodactyly & $25.0 \%$ \\
\hline Bilateral thumb sign & $18.0 \%$ \\
\hline Dental crowding and/or a high or narrow palate & $18.0 \%$ \\
\hline Atrophic scars in at least two places & $14.0 \%$ \\
\hline Mitral valve prolapse & $7.0 \%$ \\
\hline Bilateral wrist sign & $7.0 \%$ \\
\hline Aortic root dilation & $3.5 \%$ \\
\hline Arm-span-to-height ratio $\geq 1.05$ & $3.5 \%$ \\
\hline Recurrent and multiple abdominal hernias & $3.5 \%$ \\
\hline Pelvic, rectal, or uterine prolapse & $0.0 \%$ \\
\hline \multicolumn{2}{|l|}{ Frequency of spinal deformity and spine surgery } \\
\hline Scoliotic inflection & $32.0 \%$ \\
\hline Scoliosis & $29.0 \%$ \\
\hline Spine surgery & $0.0 \%$ \\
\hline
\end{tabular}

In addition, $32.1 \%$ of the patients $(n=9 / 28)$ presented with a simple scoliotic inflection and $39.3 \%(n=11 / 28)$ had neither scoliosis nor a scoliotic inflection (Table 1).

\section{DISCUSSION}

In this study, the authors evaluated the frequency and severity of scoliosis in patients with hEDS. Limited data regarding scoliosis in hEDS are currently available in the literature. Results showed that $29 \%$ of the patients with hEDS presented with scoliosis at the initial diagnosis. Scoliosis was not retained among the diagnostic criteria for hEDS in the 2017 classification. This choice could be challenged in light of other subjective criteria retained in this classification, the prevalence of which are identical or even lower. The question remains for the potential relationship between scoliosis, hypermobility, and hEDS, which was not the objective of this study.

\section{Natural History of Scoliosis and the Impact of Joint Hypermobility}

None of the patients had a severe form of scoliosis; most were benign. However, regarding natural history of scoliosis, it is critical to diagnose scoliosis early in order to ensure regular follow-up and appropriate management of hypermobile patients. 
Idiopathic scoliosis is frequent, with an overall prevalence of $1-3 \%$ most often cited in the literature. ${ }^{19}$ The female-to-male ratio ranges from 1.5:1 to 3:1 and increases substantially with increasing age. ${ }^{20}$ The prevalence of generalised joint hypermobility among children and adolescents varies significantly (7-65\%), depending on measurement method, sex, age, and ethnicity. ${ }^{21}$ Several studies suggested that generalised joint hypermobility is more common in subjects with scoliosis compared to a control population of patients of the same sex and age. A 2011 study by Czaprowski et al.,22 which observed 70 subjects with idiopathic scoliosis ( 59 girls and 11 boys), found generalised joint hypermobility in $>50.8 \%$ of girls $(n=30 / 59)$ and $54.5 \%$ of boys $(n=6 / 11)$ compared with a control group of healthy subjects (21.0\% of girls and $16.0 \%$ of boys); this difference was significant. ${ }^{22}$ A second study by Czaprowski et al. $^{9}$ in 2014 confirmed these data in 155 girls aged 9-18 years with idiopathic scoliosis compared to a control group of 201 girls without scoliosis. It was revealed that $23.2 \%$ of girls with scoliosis were hypermobile compared to $13.4 \%$ in the control group ( $p=0.02) .{ }^{9}$ However, joint hypermobility did not seem to influence the prognosis of scoliosis. In this study, there was no correlation between the presence of generalised joint hypermobility and the severity of scoliosis. There was no correlation between the measured Beighton score and the Cobb angle $(p=0.93){ }^{9}$

A 2018 study carried out among 822 Turkish students (413 boys and 409 girls) with an average age of 12.2 years (SD: \pm 1.3 years) found hypermobility in 151 subjects, i.e., $18.6 \%$ of the study population (10.2\% of girls and $8.4 \%$ of boys). Scoliosis was found in $5.2 \%$ of children $(n=43 / 822)$, of which $23.2 \% \quad(n=10 / 43)$ were hypermobile and $76.8 \%(n=33 / 43)$ were not. The presence of hypermobility was not associated with the presence of scoliosis. ${ }^{23}$

Interestingly, a study by Haller et al., ${ }^{24}$ performed in 570 women with idiopathic scoliosis, looked at whether the Beighton score was predictive of scoliosis surgery. Between the different elements of the Beighton score measure, only the inability to put hands flat on the floor was predictive of the progression of scoliosis to surgery. Generalised joint hypermobility did not influence the risk of surgery, while lack of hypermobility (a Beighton score of $0 / 9$ ) increased the risk of surgery in scoliosis. The authors specified that women who could not put their hands flat on the floor had a 2.1-times greater chance of having surgery compared to those who could $(p=0.001) .{ }^{24}$ This single measure was useful and easy to perform for predicting the progression of idiopathic scoliosis. The hypermobility and flexibility of the spine could be, according to the authors, factors of better response to the brace, avoiding aggravation and need for surgery.

\section{Scoliosis and Ehlers-Danlos Syndrome}

EDS is one of the possible diagnoses to consider in the presence of unusual or secondary appearance scoliosis. Ligament laxity, postural abnormalities, and muscle weakness found in EDS could be factors favouring this scoliosis. ${ }^{23}$ There are limited data available in the literature regarding the frequency and severity of scoliosis in the different forms of EDS. ${ }^{12}$ In kyphoscoliotic EDS, scoliosis is secondary to severe muscle hypotonia and ligament laxity." It appears early in infancy and is part of the diagnostic criteria. Molecular biology can confirm the diagnosis. In these early forms, the progression of scoliosis is severe and surgical treatment is often necessary.

In 2000, Stanitski et al. ${ }^{10}$ published a large series of patients affected by three different forms of EDS (hypermobile, classic, or vascular) in whom scoliosis was evaluated.10 Thirty-three percent of patients had scoliosis (30\% of hEDS, 33\% of vascular EDS, and $36 \%$ of classic EDS). Scoliosis therefore seems slightly more frequent in classic EDS, a feature already found in a previous publication according to the authors. No patient presented with scoliosis requiring surgery (Cobb angle $>50^{\circ}$ ). The curves were mild-to-moderate in $71 \%$ of cases with hEDS. No significant correlation was found between back pain and the existence of scoliosis. ${ }^{10}$ Analysis of EOS spine X-ray of the patients confirmed the data available in the literature. Twenty-nine percent of patients with hEDS presented with mild-to-moderate scoliosis. No patient had a severe scoliosis. Interestingly, $32 \%$ of patients presented with a scoliotic inflection, not to be confused with scoliosis. This scoliotic inflection could be linked to an external phenomenon, such as inequality in length of the lower limbs or a tilting of the pelvis.

In adulthood, only $10 \%$ of cases of mild-tomoderate scoliosis have a risk of progression; ${ }^{24}$ in theory, there is therefore very little risk of 
progression of scoliosis in the population of patients with hEDS. Even if joint hypermobility seems to influence the development of scoliosis, it seems that its presence is reassuring regarding the possible progression of scoliosis. ${ }^{24}$ It also seems to be the case in the hypermobile adult population of this study, where no severe scoliosis requiring surgery was found. During clinical examination, the ability to put hands flat on the floor, assessed by the Beighton score and found in $75 \%(n=6 / 8)$ of patients with a scoliosis, also seems to be an interesting element in the prediction of the favourable evolution of scoliosis. Further studies are needed to determine if the ability to put hands flat on the floor could be a factor explaining the good response to brace treatment in hypermobile patients with moderate or progressive scoliosis, and whether this could explain the absence of severe scoliosis in patients with hEDS.

Scoliosis was not retained among the diagnostic criteria for hEDS in the 2017 classification. This choice can be challenged in light of other subjective criteria retained in this classification, the frequency of which are identical or even lower.

\section{Study Limitations}

This present study should be considered with certain limitations. Firstly, the study was performed using a small population size and it would therefore be necessary to assess the exact prevalence of scoliosis in a larger population of patients with hEDS. Secondly, prevalence was not compared with a group of patients presenting with only joint hypermobility. Currently, it is not possible to conclude if scoliosis could be linked to hypermobility itself or if it could be considered as an additional criterion in hEDS diagnosis. A comparative study with a control group should be considered. Moreover, it is worthy of note that hEDS is mainly seen in the female population in which scoliosis is much more frequent, meaning that some scoliosis could be linked to sex and not to disease itself. ${ }^{7}$ Thirdly, absence of genetic confirmation in hEDS makes the diagnosis difficult since it is mainly based on clinical examination plus some echographic features.

Highlighting the prevalence of scoliosis in patients with hEDS, this study supports the importance of considering screening for scoliosis in any patients meeting the criteria for hEDS. Regular follow-ups could avoid scoliosis progression by appropriate management. It seems fundamental, given the preponderance of females in the hEDS population, to be attentive to screening for scoliosis at the first signs of puberty. Repeated use of EOS makes it possible to monitor scoliosis until patients are adults.

\section{CONCLUSION}

This retrospective study showed that scoliotic inflections and scoliosis are frequent in patients with hEDS. Considering this observation, the authors recommend performing full spinal X-ray in all patients with hEDS at the time of initial diagnosis and regularly during follow-up.

\section{References}

1. Castori M, Hakim A. Contemporary approach to joint hypermobility and related disorders. Curr Opin Pediatr. 2017;29(6):640-9.

2. Malfait $\mathrm{F}$ et al. The 2017 international classification of the Ehlers-Danlos syndromes. Am J Med Genet Part C Semin Med Genet. 2017;175(1):8-26.

3. Malfait $\mathrm{F}$ et al. Defective initiation of glycosaminoglycan synthesis due to B3GALT6 mutations causes a pleiotropic Ehlers-Danlos-syndromelike connective tissue disorder. Am J Hum Genet. 2013;92(6):935-45.

4. Ritelli $M$ et al. Expanding the clinical and mutational spectrum of recessive AEBP1-related classical-like EhlersDanlos syndrome. Genes (Basel).
2019;10(2):135

5. Joseph AW et al. Characteristics, diagnosis, and management of Ehlers-Danlos syndromes: a review. JAMA Facial Plast Surg. 2018;20(1):70-5.

6. Tinkle B et al. Hypermobile EhlersDanlos syndrome (a.k.a. EhlersDanlos syndrome Type III and EhlersDanlos syndrome hypermobility type): clinical description and natural history. Am J Med Genet C Semin Med Genet. 2017;175(1):48-69.

7. Demmler JC et al. Diagnosed prevalence of Ehlers-Danlos syndrome and hypermobility spectrum disorder in Wales, UK: a national electronic cohort study and case-control comparison. BMJ Open. 2019;9(11):e031365.

8. Goldstein LA, Waugh TR. Classification and terminology of scoliosis. Clin Orthop Relat Res. 1973;93:10-22.

9. Czaprowski D. Generalised joint hypermobility in caucasian girls with idiopathic scoliosis: relation with age, curve size, and curve pattern. ScientificWorldJournal. 2014;2014:370134.

10. Stanitski DF et al. Orthopaedic manifestations of Ehlers-Danlos syndrome. Clin Orthop Relat Res. 2000;(376):213-21. 
11. Brady AF et al. The Ehlers-Danlos syndromes, rare types. Am J Med Genet Part C Semin Med Genet. 2017;175(1):70-115

12. Stern $\mathrm{CM}$ et al. Musculoskeletal conditions in a pediatric population with Ehlers-Danlos Syndrome. J Pediatr. 2017;181:261-66.

13. The Ehlers-Danlos Society. hEDS diagnostic checklist. 2021. Available at: https://www.ehlers-danlos.com/ heds-diagnostic-checklist/. Last accessed: 14 January 2021

14. Bulbena $\mathrm{A}$ et al. Clinical assessment of hypermobility of joints: assembling criteria. J Rheumatol. 1992;19(1):11522.

15. Smits-Engelsman $B$ et al. Beighton score: a valid measure for generalized hypermobility in children. J Pediatr. 2011;158(1):119-23.
16. Juul-Kristensen B et al. Measurement properties of clinical assessment methods for classifying generalized joint hypermobility - a systematic review. Am J Med Genet Part C Semin Med Genet. 2017;175(1):116-47.

17. Naal FD et al. Validation of a selfreported Beighton score to assess hypermobility in patients with femoroacetabular impingement. Int Orthop. 2014;38(11):2245-50.

18. Roussouly P et al. Classification of the normal variation in the sagittal alignment of the human lumbar spine and pelvis in the standing position. Spine (Phila Pa 1976). 2005;30(3):346-53

19. Negrini $\mathrm{S}$ et al. 2016 SOSORT guidelines: orthopaedic and rehabilitation treatment of idiopathic scoliosis during growth. Scoliosis Spinal Disord. 2018;13(1):1-48.
20. Konieczny MR et al. Epidemiology of adolescent idiopathic scoliosis. J Child Orthop. 2013;7(1):3-9.

21. Adib $\mathrm{N}$ et al. Joint hypermobility syndrome in childhood. A not so benign multisystem disorder? Rheumatology (Oxford). 2005;44(6):744-50.

22. Czaprowski $D$ et al. Joint hypermobility in children with idiopathic scoliosis: SOSORT award 2011 winner. Scoliosis. 2011;6:22.

23. Bozkurt S et al. Hypermobility frequency in school children: relationship with idiopathic scoliosis, age, sex and musculoskeletal problems. Arch Rheumatol. 2019;34(3):268-73.

24. Haller $\mathrm{G}$ et al. Lack of joint hypermobility increases the risk of surgery in adolescent idiopathic scoliosis. J Pediatr Orthop Part B. 2018;27(2):152-8 